\title{
Significance of the neutrophil-to- lymphocyte ratio in predicting the response to neoadjuvant chemotherapy in extremity osteosarcoma: a multicentre retrospective study
}

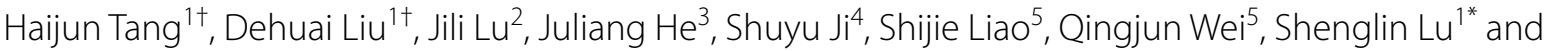 \\ Yun Liu ${ }^{6^{*}}$
}

\begin{abstract}
Background: At present, no predictive factor has been validated for the early efficacy of neoadjuvant chemotherapy (NACT) in osteosarcoma. The purpose of this study was to investigate the significance of the neutrophil-to-lymphocyte ratio (NLR) in predicting the response to NACT in extremity osteosarcoma.

Methods: Pathological complete response ( $p C R$ ) was used to assess the efficacy of NACT. Receiver operating characteristic (ROC) curves and the Youden index (sensitivity + specificity-1) were used to determine the optimal cut-off values of the NLR. Univariate and multivariate analyses using logistic regression models were conducted to confirm the independent factors affecting the efficacy of NACT.

Results: The optimal NLR cut-off value was 2.36 (sensitivity, 80.0\%; specificity, 71.3\%). Univariate analysis revealed that patients with a smaller tumour volume, lower stage, lower NLR and lower PLR were more likely to achieve $p C R$. Multivariate analyses confirmed that the NLR before treatment was an independent risk factor for $\mathrm{pCR}$. Compared to patients with a high NLR, those with a low NLR showed a more than 2-fold higher likelihood of achieving PCR (OR $2.82,95 \% \mathrm{Cl} 1.36-5.17, p=0.02)$.

Conclusion: The NLR is a novel and effective predictive factor for the response to NACT in extremity osteosarcoma patients. Patients with a higher NLR showed a lower percentage of $\mathrm{pCR}$ after NACT.
\end{abstract}

Keywords: Neutrophil-to-lymphocyte, Neoadjuvant chemotherapy, Osteosarcoma

\section{Introduction}

Osteosarcoma, which originates from mesenchymal tissue and is mainly located in the long bones, is one of the most common primary malignant tumours in children

\footnotetext{
*Correspondence: 275207660@qq.com; GXMZGK@163.com

${ }^{\dagger}$ Haijun Tang and Dehuai Liu contributed equally to this work.

1 Department of Orthopaedics, Minzu Hospital of Guangxi Zhuang

Autonomous Region, Nanning, Guangxi, China

${ }^{6}$ Department of Spine and Osteopathic Surgery, The First Affiliated

Hospital of Guangxi Medical University, Nanning, Guangxi, China

Full list of author information is available at the end of the article
}

and adolescents [1]. Before the 1970s, the main treatment method for this disease was amputation and postoperative chemotherapy. However, the prognosis of patients treated with this combination was extremely poor, and the 5 -year survival rate was approximately $42 \%$ [2]. In 1970, Cortes [3] and Rosen [4] proposed preoperative chemotherapy, which is also known as neoadjuvant chemotherapy (NACT). Since then, the survival rate has increased to $60-70 \%$, and limb salvage is possible for most patients [5]. 
According to National Comprehensive Cancer Network (NCCN) recommendations, the curative effect of chemotherapy is a key index for assessing whether limb salvage is feasible [6]. Hence, predicting the efficacy of NACT before surgery is very important. Recently, pathological complete response ( $\mathrm{pCR}$ ) has been regarded as the gold standard to estimate the efficacy of NACT [7]. However, pCR is determined based on postoperative tumour specimens and therefore cannot be validated as a predictive factor for the early efficacy of NACT. Additionally, some radiological parameters, including tumour volume, the apparent diffusion coefficient (ADC) on MRI, and standardized uptake values (SUVs) on ${ }^{18} \mathrm{~F}$-FDG PET, have also been proposed as predictive indices $[8$, 9]. Although these parameters can be measured before surgery and have relatively satisfactory reliability, measurement errors, specificity and sensitivity, and costs still need to be improved. Therefore, identifying new, reliable, and inexpensive parameters is important.

The systemic inflammatory response (SIR) has been demonstrated to play a key role in tumorigenesis, metastasis, and even drug resistance [10]. The neutrophil-tolymphocyte ratio (NLR) and platelet-to-lymphocyte ratio (PLR) are two important indices that reflect the SIR [11] and have also been studied for $\mathrm{PCR}$ prediction in various solid tumours, such as breast and oesophageal cancers $[12,13]$. Many articles have demonstrated that inflammation-based indicators, such as the NLR, PLR and CRP/ $\mathrm{Alb}$ ratio, can predict the prognosis of osteosarcoma patients [14, 15]. However, the potential prognostic value of these indicators for pCR after NACT for osteosarcoma has not been reported.

Thus, we conducted this multicentre retrospective study to determine whether the NLR or PLR can be used to predict $\mathrm{pCR}$ after NACT in osteosarcoma and therefore serve as a parameter to guide chemotherapy and surgical planning. To the best of our knowledge, this study is the first to explore the association of the response to NACT with the NLR and PLR in osteosarcoma.

\section{Patients and methods}

\section{Patients}

This multicentre study was conducted in three medical institutions. The medical records of patients diagnosed with osteosarcoma confirmed by postoperative pathology from September 2008 to December 2018 were retrospectively reviewed. The inclusion criteria were as follows: (1) patients diagnosed with osteosarcoma by postoperative histopathology; (2) patients who received standard neoadjuvant chemotherapy (NACT) before surgery; (3) patients with complete laboratory data before NACT; (4) patients who underwent tumour resection or amputation such that pathological complete response (pCR) was obtainable; and (5) patients with lesions located in the extremities. The exclusion criteria were as follows: (1) patients with infection, fever, or any blood disease; (2) patients with recurrence; and (3) patients with incomplete medical records.

The clinical and pathological stages of the tumours were defined using the Enneking classification [16]. Informed consent was obtained from all patients, and this study was supported by the Ethical Association of our institution.

\section{Blood samples and data review}

Blood samples were obtained when the patient was hospitalized to start the first cycle of chemotherapy. The NLR was calculated from routine peripheral blood examination results and defined as the absolute number of neutrophils divided by the lymphocyte count. The same formula was applied to determine the platelet-tolymphocyte ratio (PLR). Other parameters, including white blood cell count (WBC), percentage of monocytes, erythrocyte sedimentation rate (ESR), C-reactive protein (CRP) level and alkaline phosphatase (ALP) level, were also reviewed. Additionally, some clinical parameters, such as age, sex, lesion location, tumour size on imaging, and tumour subtype, were adjusted and analysed.

\section{The definition of pathological complete response}

After en block resection or amputation, pathologists evaluated the specimen and histopathologic information was obtained from the pathology report. The specimen was cut along the cross section with the largest area according to the preoperative imaging. The necrosis cell was observed by HE stain in tissue section. The necrosis rate was defined as necrotic area divided by total area. Patients whose rate were $90 \%$ or more had a pathological complete response (pCR) and patients whose rate less than $90 \%$ had a non-pCR.

\section{Regimens for NACT}

All patients received first-line NACT, and the detailed regimens were AP: doxorubicin $45 \mathrm{mg} / \mathrm{m}^{2}+$ cisplatin $75 \sim 100 \mathrm{mg} / \mathrm{m}^{2}$ and MAP: high-dose methotrexate $\quad 8 \sim 12 \mathrm{~g} / \mathrm{m} 2+$ doxorubicin $\quad 45 \mathrm{mg} / \mathrm{m}^{2}+$ cisplatin $75 \sim 100 \mathrm{mg} / \mathrm{m}^{2}$.

\section{Statistical analysis}

The mean \pm standard error and the median were used to present measurement data following a normal distribution and a skewed distribution, respectively. A receiver operating characteristic (ROC) curve and the Youden index (sensitivity + specificity-1) were used to determine the optimal cut-off values of the NLR due to the lack of a reference value in recent literature. 
We used the chi-squared test and Fisher's exact test to evaluate associations between the NLR, the PLR, other important clinical parameters, and pCR. Univariate and multivariate analyses by logistic regression models were conducted to confirm the independent factors that predict pCR. Statistical significance was considered when $p<0.05$. All data were assembled using Excel 2007, and data analyses were performed by SPSS 22.0 software.

\section{Result}

\section{Baseline data of the patients and tumours}

A total of 403 patients histopathologically diagnosed with osteosarcoma of the extremities were identified. Ultimately, 96 patients met the inclusion/exclusion criteria and were included in this study. The baseline data are shown in Table 1. Among these 96 patients, 54 were male, and the median age at diagnosis was 17 years (range 7-45 years). Eighty-four (87.5\%) tumours were located in the proximal tibia and distal femur, while 12 were located in other regions, including the proximal humerus, proximal fibula and distal tibia. The median tumour size measured on MRI was $195.37 \pm 8.74 \mathrm{~cm}^{3}$. According to the Enneking surgical staging criteria, 28 patients were classified as stage I, 46 as stage II, and 22 as stage III.

\section{The optimal cut-off values of the NLR and PLR}

As shown in Fig. 1, when pCR was defined as an end point, the areas under the curve (AUCs) for the NLR and PLR were $0.793(p=0.001)$ and $0.659(p=0.069)$, respectively. The optimal cut-off values of the NLR and PLR were 2.36 (sensitivity, 80.0\%; specificity, 71.3\%) and 115 (sensitivity, 83.3\%; specificity, 55.6\%), respectively.

Table 1 Association between clinical data and NLR/PLR

\begin{tabular}{|c|c|c|c|c|c|c|c|}
\hline \multirow[t]{2}{*}{ clinical parameters } & \multirow[t]{2}{*}{$\mathbf{n}$} & \multicolumn{3}{|l|}{ NLR } & \multicolumn{3}{|l|}{ PLR } \\
\hline & & $\begin{array}{l}\text { LNLR } \\
(n=50)\end{array}$ & $\begin{array}{l}\text { HNLR } \\
(n=46)\end{array}$ & $p$-value & $\begin{array}{l}\text { LPLR } \\
(n=30)\end{array}$ & $\begin{array}{l}\text { HPLR } \\
(n=66)\end{array}$ & $p$-value \\
\hline Age (year) & & & & 0.447 & & & 0.823 \\
\hline$\leq 17$ & 56 & 31 & 25 & & 18 & 38 & \\
\hline$>17$ & 40 & 19 & 21 & & 12 & 28 & \\
\hline Sex & & & & 0.959 & & & 0.165 \\
\hline Male & 54 & 28 & 26 & & 20 & 34 & \\
\hline Female & 42 & 22 & 20 & & 10 & 32 & \\
\hline Tumor location & & & & 0.949 & & & 0.363 \\
\hline Tibia & 40 & 28 & 12 & & 16 & 24 & \\
\hline Femur & 44 & 30 & 14 & & 10 & 34 & \\
\hline Others & 12 & 8 & 4 & & 4 & 8 & \\
\hline Tumor size $\left(\mathrm{cm}^{3}\right)$ & $195.37 \pm 8.74$ & $171.37 \pm 12.94$ & $218.34 \pm 11.24$ & 0.034 & $173.22 \pm 10.54$ & $201.97 \pm 15.24$ & 0.041 \\
\hline Enneking stage & & & & 0.024 & & & 0.321 \\
\hline I & 28 & 18 & 10 & & 10 & 18 & \\
\hline$\|$ & 46 & 26 & 20 & & 16 & 30 & \\
\hline III & 22 & 6 & 16 & & 4 & 18 & \\
\hline Subtype & & & & 0.202 & & & 0.362 \\
\hline Osteoblastic & 58 & 34 & 24 & & 15 & 43 & \\
\hline Chondroblastic & 22 & 8 & 14 & & 9 & 13 & \\
\hline Others & 16 & 8 & 8 & & 6 & 10 & \\
\hline ALP & & & & 0.013 & & & 0.763 \\
\hline Elevated & 50 & 20 & 30 & & 16 & 34 & \\
\hline Normal & 46 & 30 & 16 & & 14 & 32 & \\
\hline ESR & & & & 0.478 & & & 0.352 \\
\hline Elevated & 70 & 38 & 32 & & 20 & 50 & \\
\hline Normal & 26 & 12 & 14 & & 10 & 16 & \\
\hline CRP & & & & 0.148 & & & 0.161 \\
\hline Elevated & 64 & 38 & 26 & & 17 & 47 & \\
\hline Normal & 32 & 14 & 18 & & 13 & 19 & \\
\hline Mononuclear $\left(10^{9} / \mathrm{L}\right)$ & $0.64 \pm 0.28$ & $0.58 \pm 0.23$ & $0.66 \pm 0.29$ & 0.376 & $0.64 \pm 0.20$ & $0.65 \pm 0.31$ & 0.782 \\
\hline
\end{tabular}




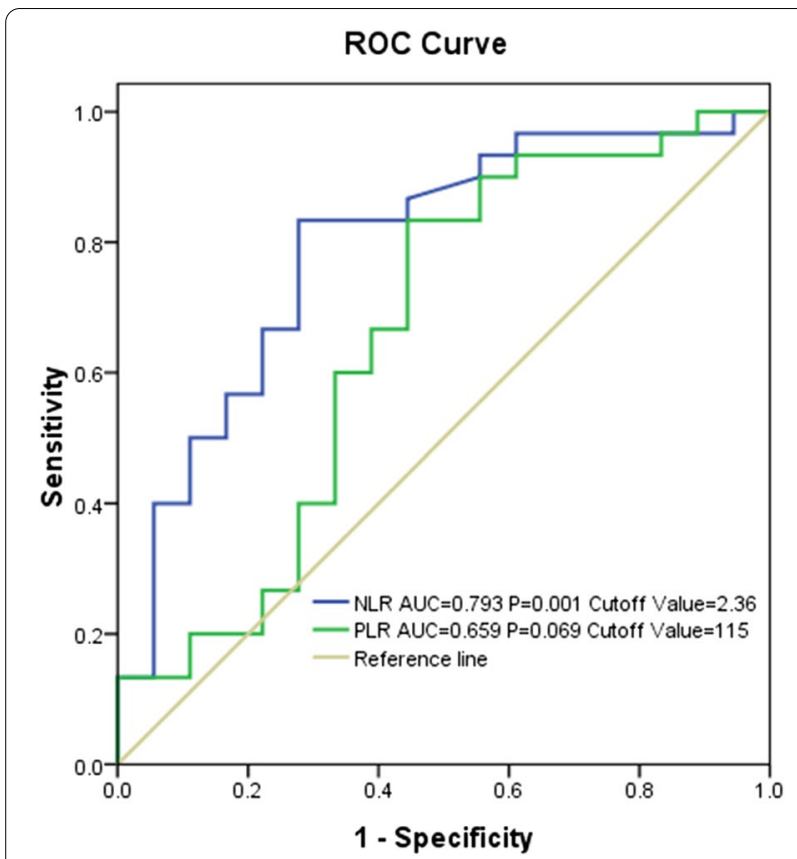

Fig. 1 The areas under the ROC curves and the optimal cut-off values of neutrophil-to-lymphocyte ratio (NLR) and platelet-to-lymphocyte ratio (PLR)

Subsequently, according to the optimal cut-off values of the NLR, patients were divided into two groups: the low NLR group (LNLR <2.36) with 50 patients and the high NLR group (HNLR $\geq 2.36$ ) with 46 patients. Similarly, 66 patients were included in the low PLR group (LPLR $<115$ ), and 30 patients were included in the high PLR group (HPLR $\geq 115$ ).

\section{Associations of the NLR and PLR with clinical parameters}

To investigate the relationships of the NLR and PLR with clinical parameters in osteosarcoma patients, comparisons between the LNLR (LPLR) and HNLR (HPLR) groups were conducted (Table 1). Our results demonstrated that the tumour size in patients with a low NLR was significantly smaller than that in patients with a high NLR $\left(171.37 \pm 12.94 \mathrm{~cm}^{3}\right.$ vs. $218.34 \pm 11.24 \mathrm{~cm}^{3}$, $p=0.034)$. A similar result was found in the PLR group $\left(173.22 \pm 10.54 \mathrm{~cm}^{3}\right.$ vs. $\left.201.97 \pm 15.24 \mathrm{~cm}^{3}, \quad p=0.041\right)$. Additionally, an advanced Enneking stage and elevated ALP were significantly associated with a high NLR, while the rates of these two parameters were not significantly different in the PLR group.

\section{Relationships between clinical parameters and pCR in univariate and multivariate analyses}

Thirty-six patients (37.5\%) achieved pCR after NACT for osteosarcoma. Univariate analysis was conducted to
Table 2 Association between patient/tumor data and pCR in univariate

\begin{tabular}{|c|c|c|c|}
\hline Variable & $\mathrm{n}$ & $\mathrm{pCR}(\%)$ & $P$ value \\
\hline Age (year) & & & 0.669 \\
\hline$\leq 17$ & 56 & $22(39.3)$ & \\
\hline$>17$ & 40 & $14(35.0)$ & \\
\hline Sex & & & 0.111 \\
\hline Male & 54 & $24(44.4)$ & \\
\hline Female & 42 & $12(28.6)$ & \\
\hline Tumor location & & & 0.896 \\
\hline Tibia & 40 & $16(40.0)$ & \\
\hline Femur & 44 & $16(36.4)$ & \\
\hline Others & 12 & $4(33.3)$ & \\
\hline Tumor size $\left(\mathrm{cm}^{3}\right)$ & & & 0.034 \\
\hline$\leq 195.37$ & 38 & $20(52.6)$ & \\
\hline$>195.37$ & 58 & 18(31.0) & \\
\hline Enneking stage & & & 0.023 \\
\hline I & 28 & 15(53.6) & \\
\hline$\|$ & 46 & $16(34.8)$ & \\
\hline III & 22 & $5(22.7)$ & \\
\hline Subtype & & & 0.263 \\
\hline Osteoblastic & 58 & $23(39.7)$ & \\
\hline Chondroblastic & 22 & $6(27.3)$ & \\
\hline Others & 16 & 7(43.8) & \\
\hline Regimens of NACT & & & 0.261 \\
\hline AP & 36 & $12(33.3)$ & \\
\hline MAP & 60 & $24(40.0)$ & \\
\hline ALP & & & 0.342 \\
\hline Elevated & 50 & $21(42.0)$ & \\
\hline Normal & 46 & 15(32.6) & \\
\hline ESR & & & 0.406 \\
\hline Elevated & 70 & $28(40.0)$ & \\
\hline Normal & 26 & $8(30.8)$ & \\
\hline CRP & & & 0.592 \\
\hline Elevated & 64 & 25(39.1) & \\
\hline Normal & 32 & $11(34.4)$ & \\
\hline NLR & & & 0.021 \\
\hline LNLR & 50 & $22(44.0)$ & \\
\hline HNLR & 46 & $14(30.4)$ & \\
\hline PLR & & & 0.031 \\
\hline LPLR & 30 & $16(53.3)$ & \\
\hline HPLR & 66 & $20(30.3)$ & \\
\hline
\end{tabular}

determine the risk factors that influenced pCR (Table 2). Our results revealed that patients with a smaller tumour volume, lower stage, lower NLR and lower PLR were more likely to achieve pCR. Subsequently, tumour size, Enneking stage, the NLR and the PLR were included in multivariate analyses. As shown in Table 3, the NLR before treatment was an independent risk factor for $\mathrm{pCR}$. 
Table 3 Association between patient/tumor characteristics and pCR in multivariate analysis

\begin{tabular}{llll}
\hline Variable & OR & $\mathbf{9 5 \% C l}$ & P value \\
\hline Tumor size $\leq 195.37$ vs $>195.37$ & 1.43 & $0.29-6.88$ & 0.65 \\
$\quad$ Enneking stage Ivs II/III & 0.98 & $0.31-3.21$ & 0.98 \\
LNLR vs HNLR & 2.82 & $1.36-5.17$ & 0.02 \\
LPLR vs HPLR & 0.73 & $0.10-5.41$ & 0.76 \\
\hline
\end{tabular}

Compared to patients with a high NLR, those with a low NLR showed a more than 2-fold higher likelihood of achieving pCR (OR 2.82, 95\% CI 1.36-5.17, $p=0.02$ ).

\section{Discussion}

In this multicentre study, we explored the predictive value of the NLR for the effectiveness of NACT in patients with osteosarcoma of the extremities. Ninety-six patients undergoing NACT treatment were included, and the NLR and PLR were calculated. We found that a low pretreatment NLR $(<2.36)$ was significantly associated with a higher rate of $\mathrm{pCR}$ and a better effect of NACT. However, other clinical parameters, including age, sex, tumour location, tumour size, clinical stage, tumour subtype, ALP, the ESR, CRP and the PLR, presented no relevance to $\mathrm{PCR}$ in our study.

Recently, many studies have demonstrated that the NLR plays a key role in predicting the treatment response to NACT and the survival rate in various cancers, such as colorectal cancer, rectal cancer, and breast cancer [17-21]. In a recent study by Chae and his colleague [22], the data of 87 patients with breast cancer were retrospectively analysed. They found that patients in the low NLR group had a higher rate of pCR than those in the high NLR group (42.1\% vs. $18.4 \%, p=0.018$, 95\% CI: 1.36-5.17). Kim et al. [8] used the pretreatment NLR and PLR as prognostic indicators of pCR in patients with gastric cancer. Kim IY et al. reported that an elevated NLR before NACT can be used as a predictor of a poor tumour response and an unfavourable prognosis in rectal cancer [19]. Our observations are consistent with those of previous studies.

Many oncologists and pathologists have suggested reasons for the association between a higher NLR in peripheral blood and tumour samples and higher-grade malignancy in cancer. Kk Liu et al. showed that an elevated NLR was significantly associated with higher pathological $\mathrm{T}$ stages and poor overall survival in bladder urothelial cancer cells [23]. The associations between an elevated NLR and an incomplete response and a poor prognosis in cancer are complex. We propose three main contributors to these associations. First, inflammatory cells in the tumour microenvironment can secrete a variety of cytokines, chemokines and cytotoxic mediators, which can induce early cell carcinogenesis and promote tumour occurrence [24]. Second, these cells can activate various downstream transcription factors, induce the expression of antiapoptotic genes and activate cyclin, thus promoting the survival and proliferation of tumour cells [25]. Moreover, inflammatory reactions can activate a variety of enzymes, which can increase the aggressiveness and metastatic potential of tumour cells by degrading extracellular matrix [26]. Neutrophils and lymphocytes are the most important cells in the inflammatory reaction and contribute to inflammation in the tumour microenvironment. Neutrophils can promote extracellular matrix reconstruction, tumour growth, metastasis and drug resistance [27-29] In addition, neutrophils promote angiogenesis by releasing vascular endothelial factors, including vascular endothelial growth factor (VEGF), thus promoting tumour invasion [30]. In contrast, lymphocyte-mediated cytotoxicity can inhibit tumour proliferation and metastasis [31]. In general, a higher NLR corresponds to a more severe systemic inflammatory response, suggesting that the tumour has greater invasiveness, higher malignancy and stronger drug resistance. Therefore, a higher NLR corresponds to a worse effect of NACT and a lower incidence of pCR.

\section{Limitation}

Although this is multicenter study, the sample size is still relatively small. Meanwhile, this is a retrospective analysis, which may bring potential bias to the results. Thus, future multicenter prospective studies are needed to validate our findings.

\section{Conclusions}

Our findings suggest that the NLR is an important factor predicting the response to NACT in extremity osteosarcoma patients. Patients with a higher NLR showed a lower percentage of $\mathrm{pCR}$ after NACT.

\section{Abbreviations}

NACT: Neoadjuvant chemotherapy; NLR: Neutrophil-to-lymphocyte ratio; pCR: Pathological complete response; ROC: Receiver operating characteristic; SIR: Systemic inflammatory response; PLR: Platelet-to-lymphocyte ratio; WBC: White blood cell count; ESR: Erythrocyte sedimentation rate; CRP: C-reactive protein; ALP: Alkaline phosphatase; AUCs: Areas under the curve; VEGF: Vascular endothelial growth factor.

\section{Acknowledgments}

The authors would like to thank all the staff of the participating departments.

\section{Authors' contributions}

THJ, LY and LDH designed the study. JSY, LSJ and LL collected the data. LSL and WQJ evaluated the data. THJ and LSL wrote the manuscript. All authors read and approved the final manuscript. 


\section{Funding}

This study has been supported by the Natural Science Foundation of Guangxi Province (grant no. 2020GXNSFAA259088), the "Medical Excellence Award" Funded by the Creative Research Development Grant from the First Affiliated Hospital of Guangxi Medical University, and the Youth Science and Technology Project of the First Affiliated Hospital of Guangxi Medical University (grant no. 201903038). The funding source played no role in the design of the study and collection, analysis, and interpretation of data and in writing the manuscript.

\section{Availability of data and materials}

All the data needed to achieve the conclusion are presented in the paper.

\section{Declarations}

\section{Ethics approval and consent to participate}

The study was approved by the Institutional Review Board (IRB) of Minzu Hospital of Guangxi Zhuang Autonomous Region, the First Affiliated Hospital of Guangxi Medical University and Affiliated Tumor Hospital of Guangxi Medical University. The need for informed consent was waived by IRB of Minzu Hospital of Guangxi Zhuang Autonomous Region, the First Affiliated Hospital of Guangxi Medical University and Affiliated Tumor Hospital of Guangxi Medical University because of the retrospective nature of the study. The study was performed in accordance with the relevant guidelines and regulations.

\section{Consent for publication}

Not applicable.

\section{Competing interests}

The authors declare that they have no competing interests.

\section{Author details}

1 Department of Orthopaedics, Minzu Hospital of Guangxi Zhuang Autonomous Region, Nanning, Guangxi, China. ²People's Hospital of Baise, Baise, Guangxi, China. ${ }^{3}$ Departments of Surgery of Bone and Soft Tissue Tumor, Affiliated Tumor Hospital of Guangxi Medical University, Nanning, Guangxi, China. ${ }^{4}$ Departments of General Practice, The First Affiliated Hospital of Guangxi Medical University, Nanning, Guangxi, China. ${ }^{5}$ Departments of Orthopedics, The First Affiliated Hospital of Guangxi Medical University, Nanning, Guangxi, China. ${ }^{6}$ Department of Spine and Osteopathic Surgery, The First Affiliated Hospital of Guangxi Medical University, Nanning, Guangxi, China.

\section{Received: 11 July 2021 Accepted: 20 December 2021}

Published online: 04 January 2022

\section{References}

1. Bielack S, Carrle D, Casali PG. Osteosarcoma: ESMO clinical recommendations for diagnosis, treatment and follow-up. Ann Oncol. 2009;20(Suppl 4):137-9.

2. Gross SW. Sarcoma of the long bones; based upon a study of one hundred and sixty-five cases. Clin Orthop Relat Res. 1975;438(156):5.

3. Cortes EP, Holland JF, Wang JJ, Glidewell O. Adriamycin and amputation in primary osteogenic sarcoma. Proc Am Assoc Cancer Res Ann Meeting. 1974;15(3):170.

4. Rosen G, Suwansirikul S, Kwon C, Tan C, Wu SJ, Murphy ML. High-dose methotrexate with citrovorum factor rescue and adriamycin in childhood osteogenic sarcoma. Cancer. 2015;33(4):1151-63.

5. Faisham WI, Mat Saad AZ, Alsaigh LN, Nor Azman MZ, Kamarul Imran M, Biswal BM. Prognostic factors and survival rate of osteosarcoma: a single-institution study. Asia-Pac J Clin Oncol. 2017;13(2):e104-10.

6. National Comprehensive Cancer Network. Bone Cancer (Version 1.2018). Accessed 29 Aug 2017.

7. Chen Y, Chen K, Xiao X, Nie Y, Qu S, Gong C. Pretreatment neutrophil-to lymphocyte ratio is correlated with response to neoadjuvant chemotherapy as an independent prognostic indicator in breast cancer patients: a retrospective study. BMC Cancer. 2016;16(1):320.
8. Kim MS, Lee SY, Cho WH, Song WS, Koh JS, Lee JA. Initial tumor size predicts histologic response and survival in localized osteosarcoma patients. J Surg Oncol. 2008;97(5):456-61.

9. De Vries AF, Kremser C, Hein PA, Griebel J, Krezcy A, Ofner D. Tumor microcirculation and diffusion predict therapy outcome for primary rectal carcinoma. Int J Radiat Oncol Biol Phys. 2003;56:958-65.

10. Balkwill FR, Mantovani A. Cancer-related inflammation: common themes and therapeutic opportunities. Semin Cancer Biol. 2012;22(1):33-40.

11. Dolan RD, McSorley ST, Horgan PG, Laird B, McMillan DC. The role of the systemic inflammatory response in predicting outcomes in patients with advanced inoperable cancer: systematic review and meta-analysis. Crit Rev Oncol Hematol. 2017;116:134-46.

12. Asano Y, Kashiwagi S, Onoda N, Noda S, Kawajiri H, Takashima T. Predictive value of neutrophil/lymphocyte ratio for efficacy of preoperative chemotherapy in triple-negative breast cancer. Ann Surg Oncol. 2016;23(4):1104-10.

13. Mclaren PJ, Bronson NW, Hart KD, Vaccaro GM, Gatter KM, Thomas CR. Neutrophil-to-lymphocyte and platelet-to-lymphocyte ratios can predict treatment response to neoadjuvant therapy in esophageal cancer. J Gastrointest Surg. 2017;21(4):607-13.

14. Yong-Jiang L, Kai Y, Min-Xun L, Wen-Biao Z, Cong X, Chong-Qi T. Prognostic value of the $C$-reactive protein to albumin ratio: a novel inflammation-based prognostic indicator in osteosarcoma. Onco Targets Ther. 2017; 10:5255-61.

15. Bangjian L, Yujing H, Yuanjue S, Jianjun Z, Yang Y, Zan S, et al. Prognostic value of inflammation-based scores in patients with osteosarcoma. Sci Rep. 2016;6:39862.

16. Enneking WF. Musculoskeletal tumor surgery. New York: Churchil Livingstone, Inc; 1983.

17. Walsh SR, Cook EJ, Goulder F, Justin TA, Keeling NJ. Neutrophil-lymphocyte ratio as a prognostic factor in colorectal cancer. J Surg Oncol. 2005;91(3):181-4.

18. Kim TG, Park W, Kim H. Baseline neutrophil-Lym- phocyte ratio and platelet-lymphocyte ratio in rectal cancer patients following neoadjuvant chemoradiotherapy. Tumori. 2018;105(5):434-40.

19. Kim IY, You SH, Kim YW. Neutrophil-lymphocyte ratio predicts pathologic tumor response and survival after preoperative chemoradiation for rectal cancer. BMC Surg. 2014;14:94.

20. Proctor MJ, McMillan DC, Morrison DS, Fletcher CD, Horgan PG, Clarke SJ. A derived neutrophil to lympho- cyte ratio predicts survival in patients with cancer. Br J Cancer. 2012;107:695-9.

21. Shen L, Zhang H, Liang L, Li G, Fan M, Wu Y. Baseline neutrophil- lymphocyte ratio ( $\geq 2.8$ ) as a prognostic factor for patients with locally advanced rectal cancer undergoing neoadjuvant chemoradiation. Radiat Oncol. 2014:9:295.

22. Chae S, Kang KM, Kim HJ, Kang E, Park SY, Kim JH. Neutrophil-lymphocyte ratio predicts response to chemotherapy in triple-negative breast cancer. Curr Oncol. 2018;25(2):e113.

23. Liu K, Zhao L, Wang ES. The prognostic values of tumor-infiltrating neutrophils, lymphocytes and neutrophil/lymphocyte rates in bladder urothelial cancer. Pathol Res Pract. 2018;214(8):1074-80.

24. Allavena P, Garlanda C, Borrello MG, Sica A, Mantovani A. Pathways connecting inflammation and cancer. Curr Opin Genet Dev. 2008;18(1):3-10.

25. Okada F. Inflammation-related carcinogenesis: current findings in epidemiological trends, causes and mechanisms. Yonago Acta Medica. 2014;57(2):65-72.

26. Grivennikov SI, Kuprash DV, Liu ZG, Nedospasov SA. Intracellular signals and events activated by cytokines of the tumor necrosis factor superfamily: from simple paradigms to complex mechanisms. Int Rev Cytol. 2006;252(undefined):129-61.

27. De Larco JE, Wuertz BRK, Furcht LT. The potential role of neutrophils in promoting the metastatic phenotype of tumors releasing interleukin-8. Clin Cancer Res. 2004;10(15):4895-900.

28. Müller I, Munder M, Kropf P. Polymorphonuclear neutrophils and T lymphocytes: strange bedfellows or brothers in arms? Trends Immunol. 2009;30(11):522-30.

29. Rodriguez PC, Ernstoff MS, Hernandez C, Atkins M, Zabaleta J, Sierra R. Arginase I-producing myeloid-derived suppressor cells in renal cell 
carcinoma are a subpopulation of activated granulocytes. Cancer Res. 2009;69(4):1553-60.

30. Dumitru CA, Lang S, Brandau S. Modulation of neutrophil granulocytes in the tumor microenvironment: mechanisms and consequences for tumor progression. Semin Cancer Biol. 2013;23(3):141-8.

31. Ownby HE. The prognostic values of tumor-infiltrating neutrophils, lymphocytes and neutrophil/lymphocyte rates in bladder urothelial cancer. Cancer. 1983;52(1):126-30.

\section{Publisher's Note}

Springer Nature remains neutral with regard to jurisdictional claims in published maps and institutional affiliations.

- fast, convenient online submission

- thorough peer review by experienced researchers in your field

- rapid publication on acceptance

- support for research data, including large and complex data types

- gold Open Access which fosters wider collaboration and increased citations

- maximum visibility for your research: over $100 \mathrm{M}$ website views per year

At BMC, research is always in progress.

Learn more biomedcentral.com/submissions 